\title{
Postoperative Paralumbar Pseudoaneurysm: A Case Report
}

\author{
Abdullah Talat Eissa ${ }^{1} \quad$ Ahmed Shahbaz $^{2} \quad$ Fahd Alhelal $^{1} \quad$ Ali Alhandi $^{1} \quad$ Majed Abaalkhail $^{1}$ \\ Sami Aleissa ${ }^{1}$ Faisal Konbaz ${ }^{1}$ \\ ${ }^{1}$ Department of Orthopedic Spine Surgery, King Abdulaziz Medical \\ City-Central Region, Riyadh, Saudi Arabia \\ 2 Department of Intervention Radiology, King Abdulaziz Medical \\ City-Central Region, Riyadh, Saudi Arabia \\ Address for correspondence Abdullah Talat Eissa, MD, Bsc, \\ Department of Orthopedic Spine Surgery, King Abdulaziz Medical \\ City-Central Region, P.O. Box 88496, Riyadh 11662, Saudi Arabia \\ (e-mail: eissaab@ngha.med.sa).
}

J Neurol Surg Rep 2020;81:e33-e37.
Abstract
Keywords
- pseudoaneurysm
- spine
- complications
- trauma
- lumbar artery

Lumbar artery pseudoaneurysm is a rare adverse event that is presented in reports. It is associated with serious injuries such as in the retroperitoneal cavity and pelvis. Furthermore, infection and iatrogenic etiology were documented as well. This case report presents a patient with a chest stab wound and a fall from a 2-m height, which resulted in multiple spinal fractures that were treated surgically. However, he presented back with lumbar artery pseudoaneurysm 2 weeks postoperatively, which was treated successfully by endovascular embolization.

\section{Introduction}

Lumbar artery pseudoaneurysm (LAPA) is an uncommon complication that is documented in limited case reports. ${ }^{1}$ Either penetrating or blunt trauma is reported to cause lumbar artery pseudoaneurysm. ${ }^{2}$ Moreover, it is accompanied by serious concomitant injuries in parts such as retroperitoneal cavity and pelvis. ${ }^{3,4}$ On the other hand, an iatrogenic etiology was documented as well after discectomy and posterior lumbar fixation with screws and cages, however it is rare and reported in limited reports. ${ }^{5}$ Furthermore, infection was found to cause lumbar artery pseudoaneurysm. ${ }^{6}$

This case report presents a patient with a chest stab wound and a fall from a 2-m height, which resulted in multiple spinal fractures that were treated surgically. However, he presented back with LAPA two weeks postoperatively, which was treated successfully by endovascular embolization.

\section{Case Presentation}

A 30-year-old male who was not known to have any previous medical illnesses was admitted under the trauma team with a case of stab wound over the anterior side of the chest and a fall from 2-m height. After applying the advanced trauma life support principles, the patient was intubated and stabilized, and then shifted to the intensive care unit(ICU). The patient had multiple injuries, with comminuted right maxillary/facial fractures, two left anterior chest stab wounds causing moderate left pneumothorax and mild left hemothorax, left anterolateral diaphragmatic hernia, with gastric and transverse colon injury, open right calcaneus fracture with intra-articular extension, lumbar spine unstable burst fracture (L2) with repulsed fragment and severe central canal stenosis, and an L5 burst fracture due to the axial loading sustained from the fall (-Fig. 1).

On admission, bilateral chest tubes were inserted followed by laparotomy by the general surgeons for primary closure of the left diaphragmatic injury, primary closure of the anterior and posterior aspects of the stomach with omentum patch, and primary closure of the transverse colon injury with omentum patch. Afterward, the patient was shifted back to the ICU in a stable condition.

The patient then underwent a second surgery for lumbar spine decompression utilizing a laminectomy of L1 and L2 with posterior instrumentation from L1 to L3, and it was decided to treat $\mathrm{L} 5$ burst fracture conservatively by brace for 6 weeks. This was chosen to avoid long segment spinal fusion from T11 to the pelvis in a young patient. After that, the patient was put in supine position and was handed to the orthopedic team to fix the calcaneal fracture. received

July 22, 2019

accepted after revision

February 25, 2020
DOI https://doi.org/

10.1055/s-0040-1710309. ISSN 2193-6358.

\footnotetext{
(c) 2020 Georg Thieme Verlag KG Stuttgart . New York
}

License terms

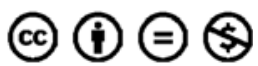



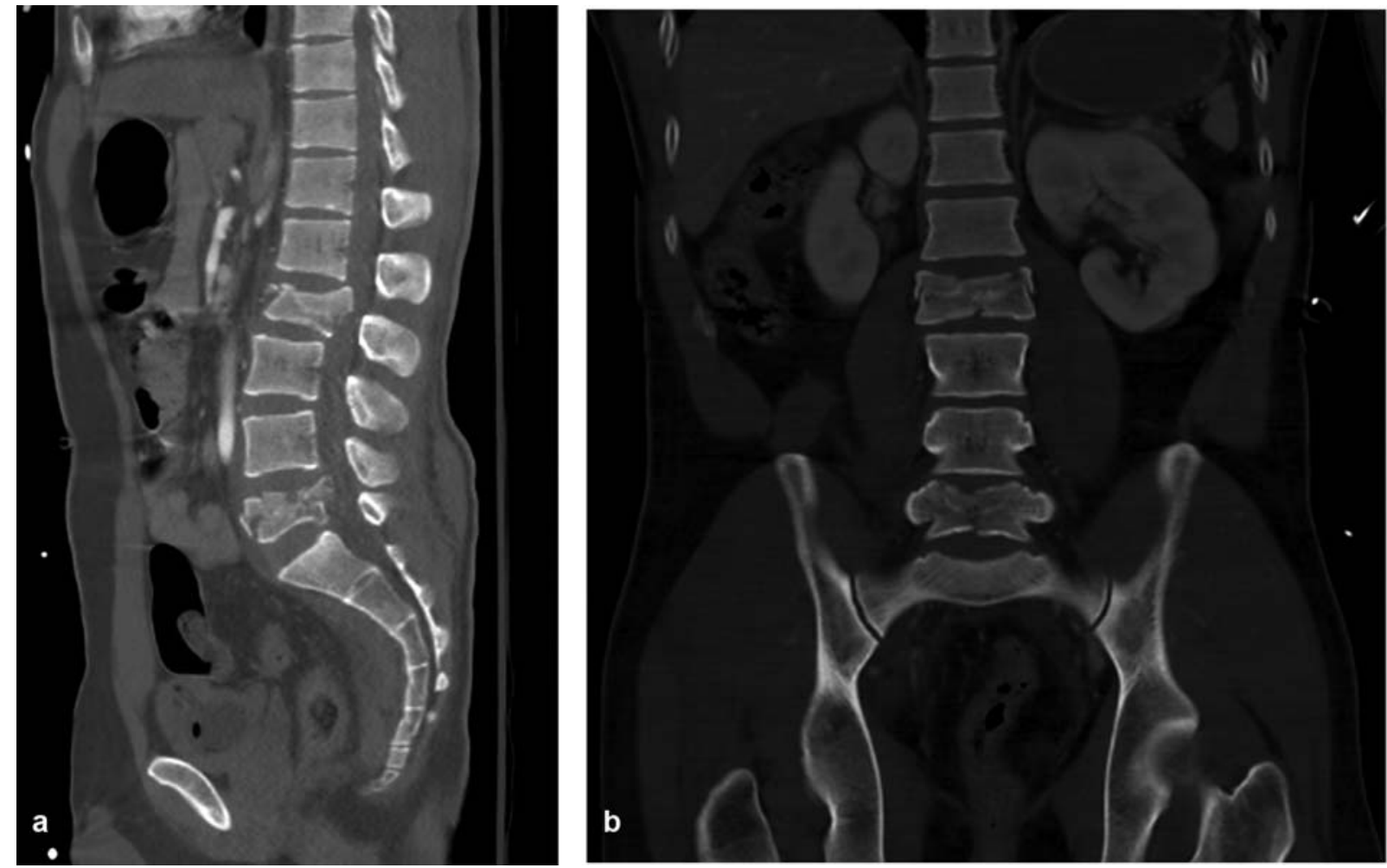

Fig. 1 The sagittal (a) and coronal (b) views of the lumbar spine CT with contrast show unstable burst fracture (L2) with repulsed fragment and severe central canal stenosis, and an L5 burst fracture. CT, computed tomography.
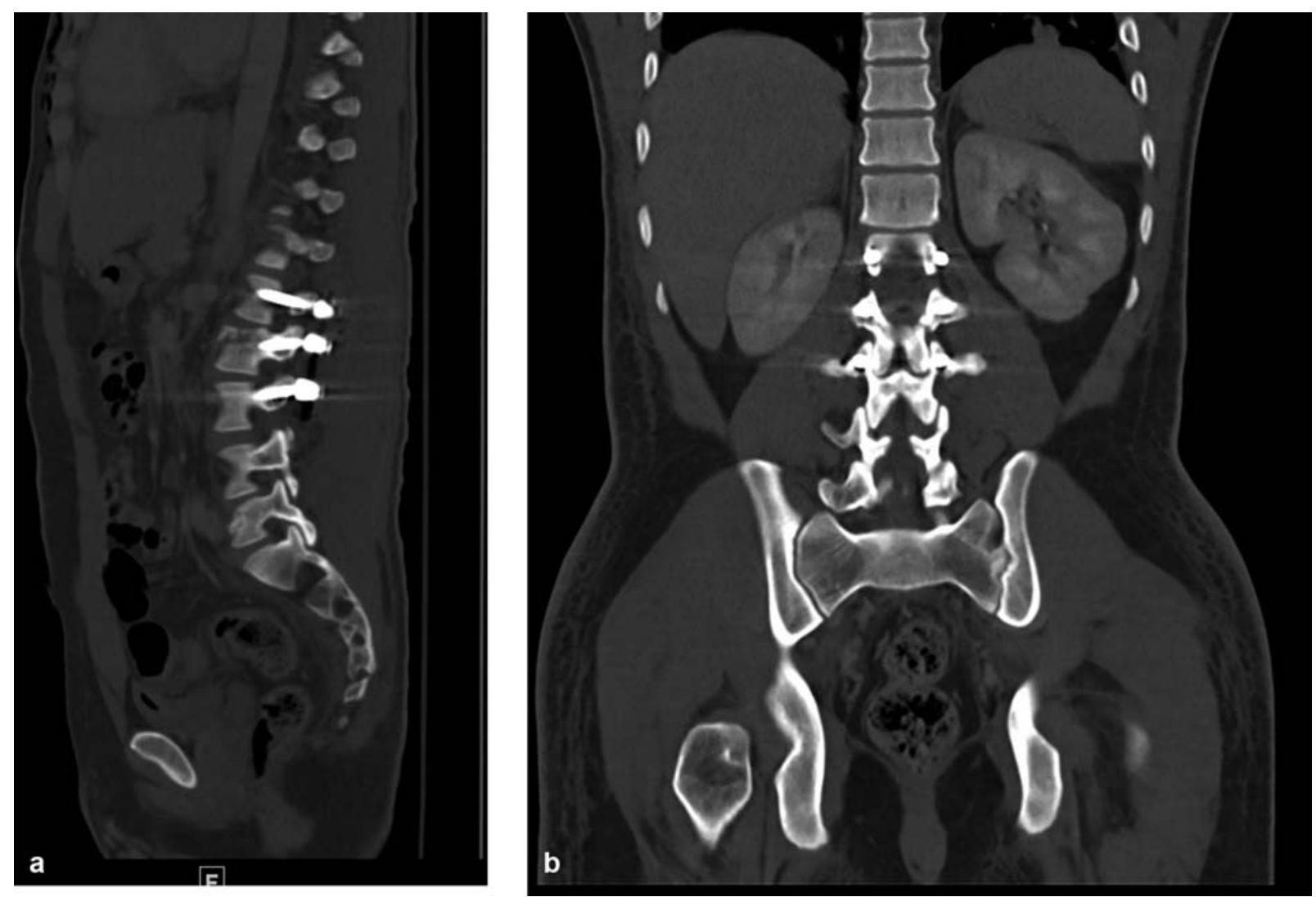

Fig. 2 The sagittal (a) and coronal (b) views of the lumbar spine CT in 4 days postoperation show intact posterior instrumentation without any complications. CT, computed tomography. 
Reconstruction of zygoma was done by the oral and maxillofacial surgery team. After extubating the patient and removing both chest tubes, he was shifted from the ICU to the ward for rehabilitation.

One week later, the lumbar spine computed tomography (CT) scan (-Fig. 2) showed an acceptable fixation and the surgical wound was healthy and intact. All medical and surgical teams agreed that the patient was suitable for discharge. Therefore, he was instructed and given all discharge plans and was discharged in a good condition.

Two weeks after the discharge, the patient came back again to the emergency room complaining of back pain and swelling with discharge from the surgical wound. He was tachycardic, afebrile, and awake and oriented. The clinical exam showed a new onset of a neurological deficit at the right L1-2 with power grade $2 / 5$, which is later found out to be due to an expanding hematoma. The patient otherwise was normal on neurological exam.

Lumbar spine CT scan was urgently done, and it showed a left paraspinous nonthrombosed actively bleeding pseudoaneurysm measuring $2.9 \times 1.7 \mathrm{~cm}$, complicated by paraspinous hematoma measuring $18 \times 4 \mathrm{~cm}$. The pseudoaneurysm was noted to be inferior to the left $\mathrm{L} 3$ pedicle screw. A left gluteal collection measuring $1.2 \times 6.5 \mathrm{~cm}$ was also noted (-Fig. 3 ).

After resuscitating the patient with intravenous fluid and two units packed red blood cells for low hemoglobin $(88 \mathrm{~g} / \mathrm{dL})$, the spine team decided to proceed with the embolization by the interventional radiology team to control the bleeding. On the same day, the patient had urgent endovascular embolization of the pseudoaneurysm.

\section{Embolization}

Right femoral artery access was obtained followed by insertion of 5-F vascular sheath. Aortic angiogram was performed which showed left paravertebral pseudoaneurysm formation arising from the level of L3/L4 on the left side (-Fig. 4). Selective lumbar artery catheterization was performed utilizing the Mickelson catheter followed by the insertion of the microcatheter. Contrast injection through

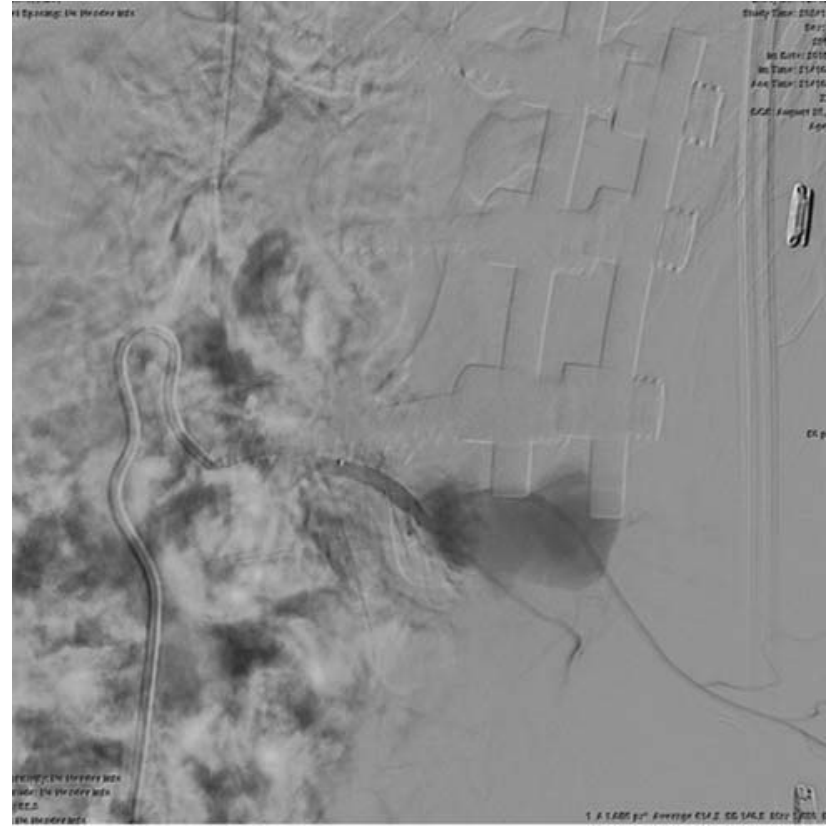

Fig. 4 Selective left L3 lumbar artery angiogram shows large lobulated pseudoaneurysm arising from lumbar artery.

microcatheter showed the pseudoaneurysm formation with active extravasation to the skin. Embolization of the pseudoaneurysm was performed utilizing Onyx (-Fig. 5). Postembolization-contrast injection showed no flow or extravasation from the pseudoaneurysm. Catheter and sheath were removed, and hemostasis was achieved with closure device.

Afterward, patient was followed and observed daily by the spine team for his vitals and serial hemoglobin in the ward. Tazocin was started as samples taken from the back hematoma returned positive Staphylococcus aureus which was sensitive to Tazocin. He was stable with no evidence of drop of hemoglobin over time. After 5 days of embolization, he was noted to have surgical wound dehiscence (-Fig. 6). To treat this, it was decided to take him for surgical irrigation and debridement.
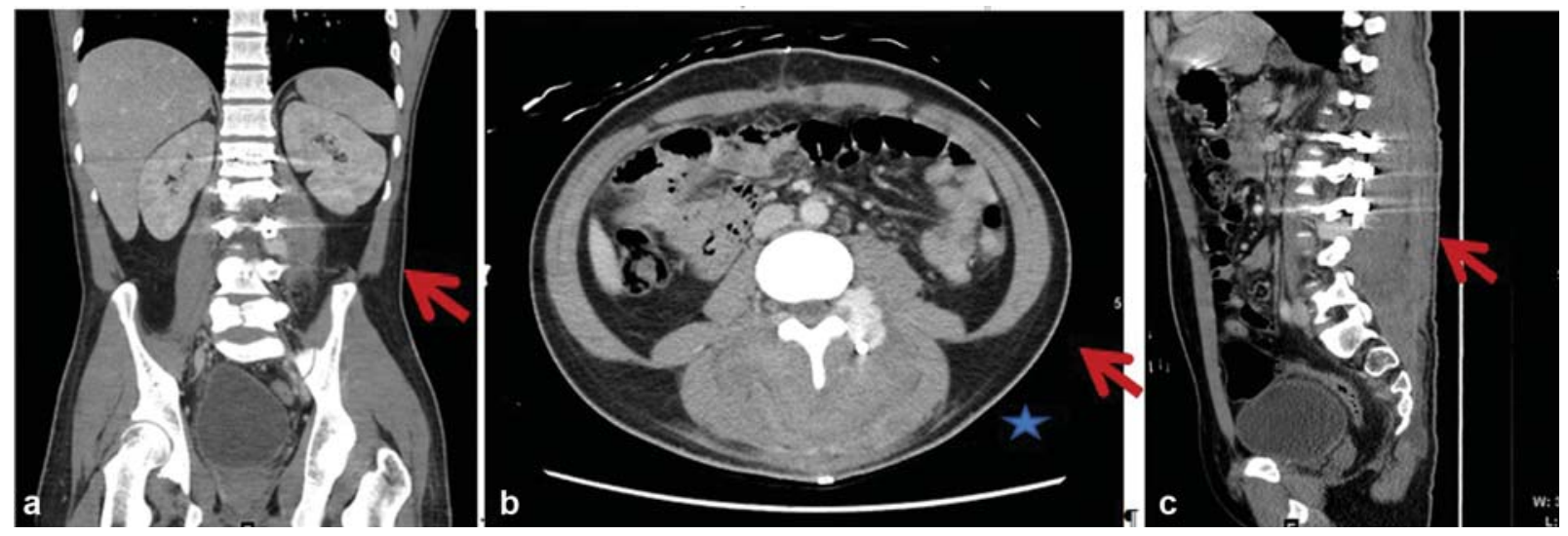

Fig. 3 The coronal (a), axial (b), and sagittal (c) views of the lumbar spine CT show left paraspinous nonthrombosed actively bleeding pseudoaneurysm (red arrow) which was noted to be inferior to the left $\mathrm{L} 3$ pedicle screw, and it was complicated by paraspinous hematoma (blue star). CT, computed tomography. 


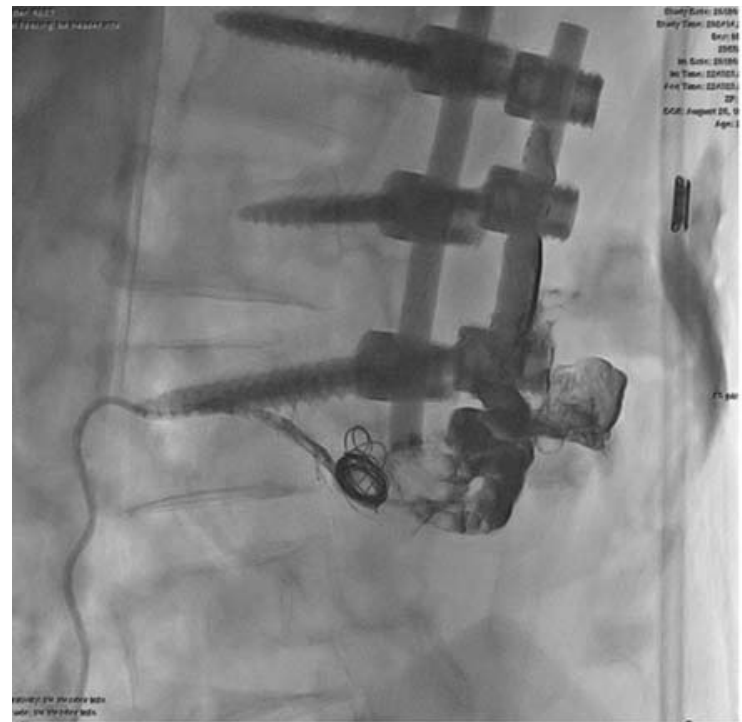

Fig. 5 Pseudoaneurysm is packed with onyx and metallic interlock embolization coil. No vascular flow shows complete occlusion.

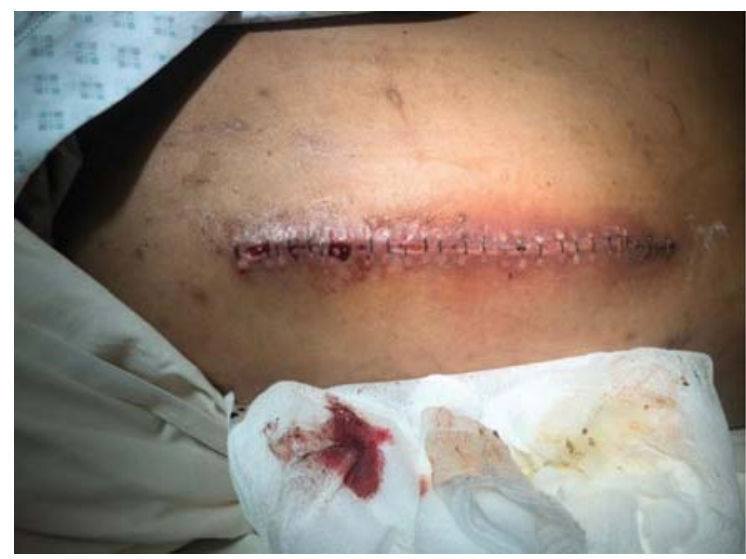

Fig. 6 Surgical wound dehiscence was noted after 5 days of embolization.

\section{Procedure Details}

Under general anesthesia the wound was opened through the previous surgical incision. There was a large hematoma which was completely removed. On the left lateral side posteriorly, the pseudoaneurysm measuring $5 \times 6 \mathrm{~cm}$ could be clearly seen ( - Fig. 7). We made sure it was completely protected. There was no active bleeding after thorough irrigation and debridement of the wound.

Intraoperative consultation to the vascular surgery team was made and it was advised not to touch the pseudoaneurysm, as it will resolve on its own since it is embolized and not pulsatile. Closure was done in layers over the drains. Dressing was applied in the usual fashion. The estimated blood loss was $300 \mathrm{~mL}$ and two Jackson-Pratt drains were applied.

Afterward, the patient was observed daily. He maintained an uneventful postoperative course with a significant improvement clinically and neurologically. He was discharged on day 13 postoperatively in an acceptable condition.

\section{Discussion}

Iatrogenic lumbar artery pseudoaneurysms remain an uncommon event after spinal procedures. The current paper reports the occurrence of such rare complication 3 weeks after spinal instrumentation for a lumbar fracture, in the context of a polytrauma patient. Previous reports of iatrogenic lumbar artery injury postspinal instrumentation described an accelerated clinical course of several hours postsurgery that may lead to hemodynamic shock and death. ${ }^{7,8}$ In our knowledge, there is only one previous case report from China, and what is unique about this report is the actual intraoperative figures, and there were no previous intraoperative pictures of lumbar spine pseudoaneurysm.

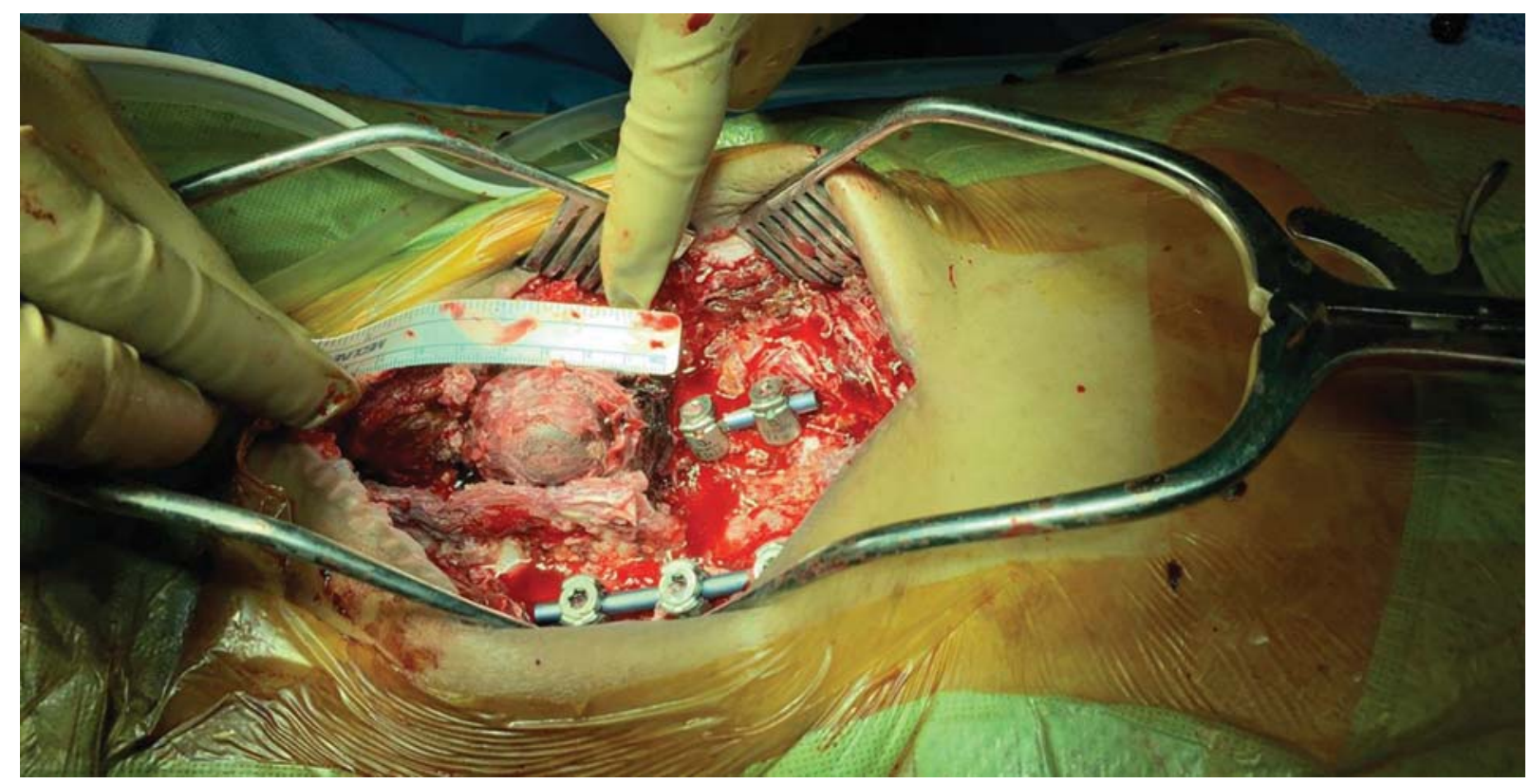

Fig. 7 Huge pseudoaneurysm measuring $5 \times 6 \mathrm{~cm}$ was noted intraoperatively. 
Development of LAPA postspinal instrumentation, however, has a slower presentation. A recent report by Latka et al showed a delayed presentation of LAPA with gradual neurological impairment postspinal instrumentation, but contrary to our patient, it was without any hemodynamic disturbances. ${ }^{9}$

The current case highlights a unique presentation in the context of a polytrauma patient of a LAPA, with blood oozing from the surgical wound, and a deep-seated infection, 2-weeks postdischarge. The patient was hemodynamically unstable and was deteriorating neurologically. Utilizing the correct diagnostic modalities and promptly intervening after diagnosing the LAPA was paramount to this patient's chances of survival. Even with the presence of an infection, endovascular embolization of the pseudoaneurysm proved to be effective in preventing further detrimental complications.

Iatrogenic vascular injury in spinal surgery may not be as rare an occurrence as it was once thought to be. A recent study by Liu et al found 20 of such reports in the literature. The report included 26 patients, 12 of which were complicated with LAPA during their clinical course. ${ }^{10}$ Survival rate was high in the paper even when accounting for two deaths out of the total 26 patients included. Further inspection of the data reveals that one patient died due to family refusal of further interventions and the other died during surgery due to shock. ${ }^{11,12}$ The report by Liu et al further emphasizes the importance of immediate intervention when the diagnosis is made. Moreover, it shows that arterial embolization (percutaneous or endovascular) was successful in all cases where it was attempted (22 patients). ${ }^{10}$

The current report showcases the ambiguity of presentation of iatrogenic LAPA, especially when accompanied by other pathologies and continues the trend of treatment success by arterial embolization.

\section{Conflict of Interest}

None.

\section{References}

1 Inamasu J, Guiot BH. Vascular injury and complication in neurosurgical spine surgery. Acta Neurochir 2006;148(04): 375-387

2 Counihan M, Pontell ME, Selvan B, Trebelev A, Nunez A. Delayed presentation of a lumbar artery pseudoaneurysm resulting from isolated penetrating trauma. J Surg Case Rep 2015;2015(07): rjv083

3 Chan KT, Korivi N. Lumbar artery pseudoaneurysm in traumatic spinal cord injury: a case report. Arch Phys Med Rehabil 2003;84 (03):455-457

4 Kessel BJ, Habib FA, Thompson B, Bajayo DE, Shatz D. Lumbar artery pseudoaneurysm. Eur J Trauma 2004;30(03):187-190

5 Nijenhuis RJ, Sluzewski M, van Rooij WJ. Iatrogenic lumbar pseudoaneurysm causing dural sac compression after spine surgery. J Neurosurg Spine 2009;10(06):585-586

6 Tomescot A, Dallaudiere B, Zurlinden O, Manelfe J. Lumbar artery pseudoaneurysm as a late complication of osteomyelitis with vertebral body destruction. J Vasc Surg 2013;58(04): 1084-1087

7 Lee KH, Park JH, Chung JW, Han JK, Shin SJ, Kang HS. Vascular complications in lumbar spinal surgery: percutaneous endovascular treatment. Cardiovasc Intervent Radiol 2000;23(01):65-69

8 Sandri A, Regis D, Marino MA, Puppini G, Bartolozzi P. Lumbar artery injury following posterior spinal instrumentation for scoliosis. Orthopedics 2011;34(04):

9 Latka K, Zurawel R, Maj B, Olbrycht T, Chowaniec J, Latka D. Iatrogenic lumbar artery pseudoaneurysm after lumbar transpedicular fixation: case report. SAGE Open Med Case Rep 2019; 7:317

10 Liu L, Li N, Wang Q, et al. Iatrogenic lumbar artery injury in spine surgery: a literature review. World Neurosurg 2019; 122:266-271

11 Ntourantonis D, Tsekouras V, Korovessis P. Delayed fatal lumbar artery bleeding following less invasive posterolateral decompression and fusion. Spine 2018;43(16):E976-E979

12 Ventura F, Barranco R, Bernabei C, Castelletti L, Castellan L. A fatal and unusual iatrogenic fourth right lumbar artery injury complicating wrong-level hemilaminectomy: a case report and literature review. Br J Neurosurg 2019;33:434-436 